\title{
Upregulation of circ_001569 predicts poor prognosis and promotes cell proliferation in non-small cell lung cancer by regulating the $\mathrm{Wnt} / \boldsymbol{\beta}$-catenin pathway
}

\author{
LINGCHI DING, WEIDONG YAO, JUNGUO LU, JUN GONG and XIAODONG ZHANG \\ Department of Oncology, Nantong Tumor Hospital, Nantong, Jiangsu 226361, P.R. China
}

Received June 28, 2017; Accepted January 5, 2018

DOI: $10.3892 / \mathrm{ol} .2018 .8673$

\begin{abstract}
Circular RNAs (circRNAs) are a large class of RNAs that have previously been identified to be involved in certain diseases, including the development of cancer. However, the role of circ_001569 in non-small cell lung cancer (NSCLC) remains unknown. In the present study, it was demonstrated that expression levels of circ_001569 were significantly increased in NSCLC tissues compared with in adjacent normal tissues. Increased circ_001569 expression was closely associated with tumor differentiation, lymph node metastasis and Tumor-Node-Metastasis classification in NSCLC. Patients that exhibited higher circ_001569 expression demonstrated a poorer survival outcome compared with patients with lower circ_001569 expression. Functional assay results indicated that the knockdown of circ_001569 inhibited the cell proliferation ability of NSCLC in vitro. In addition, it was identified that circ_001569 knockdown reduced the mRNA and protein expression levels of Wnt/ $\beta$-catenin pathway-associated genes proto-oncogene protein Wnt1, transcription factor 4 and $\beta$-catenin in NSCLC cells. Therefore, the results indicated that circ_001569 promoted cell proliferation by regulating the $\mathrm{Wnt} / \beta$-catenin pathway in NSCLC, and circ_001569 may be a potential target of NSCLC treatment.
\end{abstract}

\section{Introduction}

Lung cancer is one of the most common types of tumor and the leading cause of cancer-associated mortality in the United States in 2015 (1). Non-small cell lung cancer (NSCLC) accounts for $\sim 80 \%$ of all incidents lung cancer cases (2). Despite various improved treatments, including surgery, radiotherapy

Correspondence to: Dr Xiaodong Zhang, Department of Oncology, Nantong Tumor Hospital, 30 Tongyang North Road, Pingchao, Tongzhou, Nantong, Jiangsu 226361, P.R. China

E-mail: zhangxiaodongh1@163.com

Key words: circular RNA, circ_001569, prognosis, cell proliferation, Wnt $/ \beta$-catenin pathway and chemotherapy, the 5-year overall survival rate of patients with NSCLC is $\sim 15 \%$ (3). Therefore, investigating potential target genes for NSCLC treatment is vital.

Circular RNAs (circRNAs) are a novel type of universal and endogenous RNA. Unlike linear RNAs, circRNAs form a covalently closed continuous loop without $5^{\prime}$ or $3^{\prime}$ ends $(4,5)$. Accumulating evidence indicates that the aberrant expression of circRNAs is involved in tumor development and progression. The circular RNA ciRS-7 serves as a risk factor for hepatic microvascular invasion in hepatocellular carcinoma (6); hsa_circ_0005075 may participate in cell adhesion during hepatocellular carcinoma (HCC) development and function as a potential HCC biomarker (7); hsa_circ_0001649 expression is significantly downregulated in HCC tissues and is correlated with tumor size and the occurrence of tumor embolus in HCC (8); and circ_001569 serves as a miRNA sponge to suppress miR-145 expression, subsequently increasing transcription factor E2F5, BAG family molecular chaperone regulator 4 and formin-like protein 2 expression levels to promote the proliferation and invasion of colorectal cancer (9). To the best of our knowledge, the role of circ_001569 in NSCLC progression and its underlying mechanism have not been investigated.

In the present study, it was identified that the levels of circ_001569 were significantly increased in NSCLC tissues compared with in adjacent normal tissues. Knockdown of circ_001569 inhibited cell proliferation in NSCLC. In addition, downregulation of circ_001569 suppressed the Wnt/ $\beta$-catenin pathway in NSCLC cells. Therefore, these results indicated circ_001569 may be a potential target of NSCLC treatment.

\section{Materials and methods}

Clinical tissue specimens. A total of 56 pairs of NSCLC and adjacent normal tissues were obtained from patients (41 male cases and 15 female cases; mean age, 51.22 years; range, 38-70 years) who had undergone surgery at the Nantong Tumor Hospital (Nantong, China) between April 2011 and December 2015. The tissues were immediately snap-frozen in liquid nitrogen and stored at $-80^{\circ} \mathrm{C}$. No patient had received radiotherapy or chemotherapy prior to surgery. The Ethics Board of Nantong Tumor Hospital approved the study. Written informed consent was obtained from all patients. All patients with NSCLC were staged according to the 7 th edition of the 
American Joint Committee on Cancer Tumor-Node-Metastasis (TNM) staging system for lung cancer (10).

Cell culture. A total of 4 human NSCLC H460, H1299, SPC-A1 and A549 cell lines and the non-tumorigenic bronchial epithelium BEAS-2B cell line were purchased from the Type Culture Collection of the Chinese Academy of Sciences (Shanghai, China). Cells were cultured in RPMI-1640 medium (Life Technologies; Thermo Fisher Scientific, Inc., Waltham, MA, USA) supplemented with $10 \%$ fetal bovine serum (FBS; Life Technologies; Thermo Fisher Scientific, Inc.), 100 U/ml penicillin and $100 \mu \mathrm{g} / \mathrm{ml}$ streptomycin in a $5 \% \mathrm{CO}_{2}$ humidified atmosphere at $37^{\circ} \mathrm{C}$.

Cell transfection with small interfering (si)RNAs. The hsa-circ_001569 siRNA (si-circ_001569: 5'-GCATCG TGCAGGACTGGAA-3') and negative control (NC) were purchased from Guangzhou RiboBio Co., Ltd (Guangzhou, China), and used according to the manufacturer's protocol. When cells reached $80-90 \%$ confluency, they were transfected with 20 nM hsa-circ_001569 siRNA and negative control. Cells were transfected with Lipofectamine ${ }^{\circledR} 2000$ (Invitrogen; Thermo Fisher Scientific, Inc.), in accordance with the manufacturer's protocol. At $48 \mathrm{~h}$ post transfection, the cells were harvested and used in subsequent analysis.

$R N A$ extraction and reverse transcription-quantitative $P C R$ (RT-qPCR). Total RNA was extracted from tissues and cells using TRIzol ${ }^{\circledR}$ reagent (Life Technologies; Thermo Fisher Scientific, Inc.), according to the manufacturer's protocol. Reverse transcription was performed using $10 \mathrm{ng}$ total RNA and a PrimeScript ${ }^{\mathrm{TM}}$ RT Reagent kit (Takara Biotechnology Co., Ltd., Dalian, China). RT-qPCR was performed using an Applied Biosystems PRISM 7900HT Fast Real-Time PCR System (Thermo Fisher Scientific, Inc.) with SYBR ${ }^{\circledR}$ Premix Ex Taq ${ }^{\mathrm{TM}}$ II (Takara Bio, Inc., Otsu, Japan) with the following conditions: $50^{\circ} \mathrm{C}$ for $2 \mathrm{~min} ; 95^{\circ} \mathrm{C}$ for $10 \mathrm{~min}$; and 45 cycles of $95^{\circ} \mathrm{C}$ for $15 \mathrm{sec}$ and $60^{\circ} \mathrm{C}$ for $60 \mathrm{sec}$. GAPDH was used as a normalization control. The $2^{-\Delta \Delta \mathrm{Cq}}$ method was used to analyze relative mRNA expression (11). The primer sequences were as follows: circ_001569 forward, 5'-TCCCCTGAACATTCT CCCCAT-3'; circ_001569 reverse, 5'-GAAAGCACTTGG TGAAGTCGG-3'; GAPDH forward, 5'-CACCGTAGCCTT CCGAGTA-3'; and GAPDH reverse, 5'-GCCCTTGATGAG CTGTTGA-3'.

Cell proliferation assay. For this assay, $5 \times 10^{3}$ cells/well, transfected with si-NC or si-circ_001569 for $24 \mathrm{~h}$ at $37^{\circ} \mathrm{C}$, were seeded into 96 -well plates. Cell proliferation was examined at the indicated time points, $0,24,48$ and $72 \mathrm{~h}$, using a CCK-8 assay (Dojindo Molecular Technologies, Kumamoto, Japan), according to the manufacturer's protocol. The absorbance was read at a wavelength of $450 \mathrm{~nm}$ using a microplate reader (Molecular Devices, LLC, Sunnyvale, CA, USA).

Cell colony formation assay. A cell colony assay was performed using 6-well plates. Briefly, 200 cells/well in RPMI-1640 containing 10\% FBS were seeded into 6-well plates. Following culture in a $5 \% \mathrm{CO}_{2}$ humidified atmosphere at $37^{\circ} \mathrm{C}$ for 14 days, cells were fixed in $100 \%$ methanol at room temperature, stained with $0.5 \%$ crystal violet for $20 \mathrm{~min}$ at room temperature, and then the number of colony-forming cells were calculated using an AID iSpot Reader (Autoimmun Diagnostika GmbH, Strassberg, Germany). Three independent experiments were performed.

Western blot analysis. Total protein was extracted from cells using radioimmunoprecipitation assay lysis buffer (Beyotime Institute of Biotechnology Co., Ltd., Haimen, China). Protein concentrations were determined using a Bicinchoninic Acid Protein Assay kit (Beyotime Institute of Biotechnology, Beijing, China). Equal amounts of protein (40 $\mathrm{ng}$ ) were separated by $10 \%$ SDS-PAGE and transferred onto the nitrocellulose membranes (EMD Millipore, Billerica, MA, USA). The membranes were blocked with $5 \%$ skim milk for $2 \mathrm{~h}$ at room temperature, and then incubated with primary antibodies against proto-oncogene Wnt1 (WNT1; cat. no. sc-514531; $1: 1,000), \beta$-catenin (cat. no. sc-376841; 1:2,000), transcription factor 4 (TCF4; cat. no. sc-271287; 1:500) (all from Santa Cruz Biotechnology, Inc., Dallas, TX, USA) and GAPDH (1:1,000, cat. no. 2118; Cell Signaling Technology, Inc., Danvers, MA, USA) overnight at $4^{\circ} \mathrm{C}$. The blots were then incubated with horseradish peroxidase-conjugated goat anti-rabbit secondary antibodies (dilution, 1:2,000; cat. no. A21020; Abbkine, Wuhan, China). for $2 \mathrm{~h}$ at room temperature and visualized using an Enhanced Chemiluminescence Plus kit (GE Healthcare Bio-Sciences, Pittsburgh, PA, USA). The intensity of the bands was quantified using Image $\mathrm{Lab}^{\mathrm{TM}}$ Software (version 4.6.9; Bio-Rad Laboratories, Inc., Hercules, CA, USA).

Statistical analysis. Statistical analysis was performed using SPSS v.20.0 software (IBM Corp., Armonk, NY, USA). All data are expressed as the mean \pm standard deviation. The experiments were repeated $\geq 3$ times. Differences between two groups were assessed using the Student's t-test (two-tailed) and differences among multiple groups were analyzed using one-way analysis of variance (ANOVA). The Student-Newman-Keuls test was used as a post-hoc test following ANOVA. The associations between clinical variables and circ_001569 expression were analyzed using the Pearson $\chi^{2}$ test. Survival curves were plotted using the Kaplan-Meier method and assessed with a log-rank test to identify significant differences. $\mathrm{P}<0.05$ was considered to indicate a statistically significant difference.

\section{Results}

Circ_001569 expression is higher in NSCLC tissues and cells. To investigate the expression pattern of circ_001569 in 56 pairs of NSCLC and adjacent normal tissues, RT-qPCR assays were performed. As demonstrated in Fig. 1A, the results indicated that circ_001569 expression was significantly upregulated compared with in the adjacent normal tissues $(\mathrm{P}<0.001)$. Additionally, circ_001569 expression was significantly upregulated in the 4 human NSCLC H460, H1299, SPCA-1 cell lines and in the A549 cells, when compared with in the non-tumorigenic bronchial epithelium BEAS-2B cell line (Fig. 1B). The mean expression of circ_001569 in tumor tissues was used for cut-off levels to divide patients into the higher and lower expression groups. As determined by $\chi^{2}$ test analysis, and as summarized in Table I, circ_001569 expression 
Table I. Association between circ_001569 expression and the clinicopathological factors of patients with non-small cell lung cancer.

\begin{tabular}{|c|c|c|c|c|}
\hline \multirow[b]{2}{*}{ Clinicopathological factors } & \multirow[b]{2}{*}{ Patients (n) } & \multicolumn{2}{|c|}{ Circ_001569 expression } & \multirow[b]{2}{*}{ P-value } \\
\hline & & Lower (n=27) & Higher $(n=29)$ & \\
\hline Age & & & & 0.877 \\
\hline$\leq 60$ & 42 & 20 & 22 & \\
\hline$>60$ & 14 & 7 & 7 & \\
\hline Sex & & & & 0.643 \\
\hline Male & 41 & 19 & 22 & \\
\hline Female & 15 & 8 & 7 & \\
\hline Smoking & & & & 0.106 \\
\hline Yes & 29 & 17 & 12 & \\
\hline No & 27 & 10 & 17 & \\
\hline Differentiation & & & & $0.035^{\mathrm{a}}$ \\
\hline High and moderate & 38 & 22 & 16 & \\
\hline Lower & 18 & 5 & 13 & \\
\hline Tumor size, $\mathrm{cm}$ & & & & 0.188 \\
\hline$<3$ & 20 & 12 & 8 & \\
\hline$>3$ & 36 & 15 & 21 & \\
\hline Histology style & & & & 0.301 \\
\hline Squamous carcinoma & 20 & 7 & 13 & \\
\hline Adenocarcinoma & 24 & 14 & 10 & \\
\hline Other type & 12 & 6 & 6 & \\
\hline Lymph node metastasis & & & & $0.012^{\mathrm{a}}$ \\
\hline No & 34 & 21 & 13 & \\
\hline Yes & 22 & 6 & 16 & \\
\hline TNM classification & & & & $0.010^{\mathrm{a}}$ \\
\hline I-II & 36 & 22 & 14 & \\
\hline III & 20 & 5 & 15 & \\
\hline
\end{tabular}

${ }^{\mathrm{a}} \mathrm{P}<0.05$. TNM, Tumor-Node-Metastasis.

levels were demonstrated to be closely associated with tumor differentiation $(\mathrm{P}=0.035)$, lymph node metastasis $(\mathrm{P}=0.012)$ and TNM classification $(\mathrm{P}=0.010)$ in NSCLC. Patients who exhibited higher circ_001569 expression exhibited a poor survival outcome, compared with lower circ_001569 expression (log rank=10.641; $\mathrm{P}<0.001$; Fig. 1C).

Downregulation of circ_001569 inhibits cell proliferation of NSCLC. To additionally explore the function of circ_001569 in the development of NSCLC, loss-of-function assays were performed. siRNA was used to silence circ_001569 expression in A549 and H1299 cells, as these two cell lines exhibited higher circ_001569 expression levels when compared with H460 and SPC-A1 cells (Fig. 1D). Following transfection of the A549 and H1299 cells with si-circ_001569 at 48 and $72 \mathrm{~h}$, the CCK-8 cell proliferation assay results revealed that the proliferation rate was significantly reduced compared with that of the si-NC group (Fig. 2A and B). Following transfection of the A549 and H1299 cells with si-circ_001569 at 14 days, the cell colony formation assay results observed that the number of cell colonies was significantly reduced compared with the si-NC group (Fig. 2C and D). Therefore, the results indicated that the downregulation of circ_001569 inhibited NSCLC cell proliferation.

Reduced circ_001569 expression inhibits the Wnt/ $\beta$-catenin pathway in NSCLC cells. The Wnt/ $\beta$-catenin pathway is associated with cell proliferation and invasion in NSCLC; miRNA-148a serves as a prognostic factor, and suppresses cell migration and invasion through WNT1 in NSCLC (12). The association between circ_001569 expression and the Wnt/ $\beta$-catenin pathway in NSCLC was additionally examined. RT-qPCR analysis indicated that the knockdown of circ_001569 reduced the mRNA expression levels of WNT1, $\beta$-catenin and TCF4 in A549 and H1299 cells, as compared with in the control group (Fig. 3A and B). Consistently, it was also demonstrated that the knockdown of circ_001569 reduced the protein expression levels of WNT1, $\beta$-catenin and TCF4 in A549 and H1299 cells, as compared with in the control group (Fig. 3C and D). Therefore, these results indicated that 
A
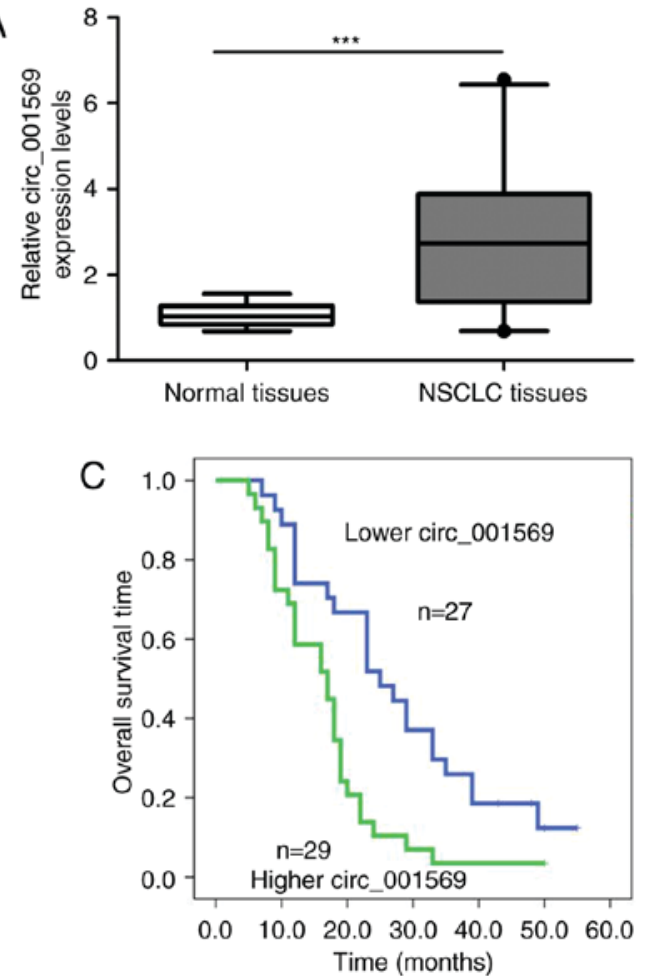

B

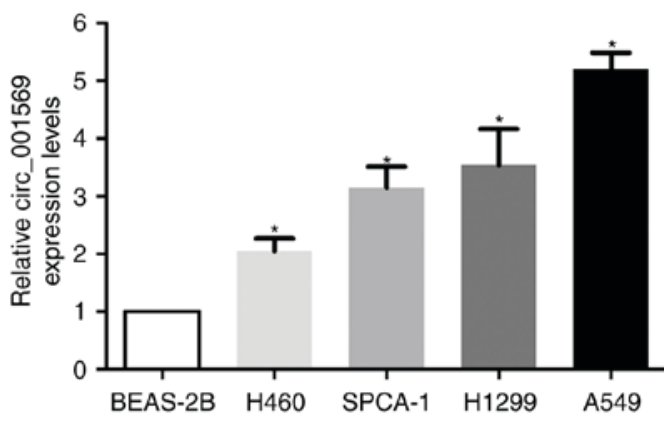

D

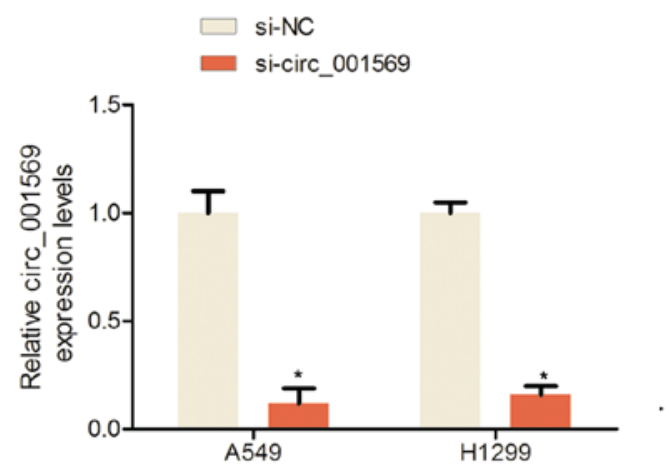

Figure 1. Expression of circ_001569 was significantly upregulated in NSCLC tissues and cells. (A) Expression of circ_001569 was upregulated in NSCLC tissues compared with in adjacent normal tissues $(\mathrm{n}=56),{ }^{* * * *} \mathrm{P}<0.001$. (B) Expression of circ_001569 was upregulated in human NSCLC H460, H1299, SPCA-1 and A549 cell lines when compared with that in the non-tumorigenic bronchial epithelium cell line BEAS-2B, ${ }^{*} \mathrm{P}<0.05$. (C) Patients who exhibited higher circ_001569 expression also exhibited a poorer survival outcome, compared with patients with lower circ_001569 expression, as determined via Kaplan-Meier analysis and the log rank test. (D) Expression of circ_001569 was measured by reverse transcription quantitative polymerase chain reaction analysis following the introduction of si-NC or si-circ_001569 into A549 and H1299 cells compared to si-NC groups, ${ }^{\text {P }}<<0.05$. NSCLC, non-small cell lung cancer; si, small interfering; $\mathrm{NC}$, negative control.

A

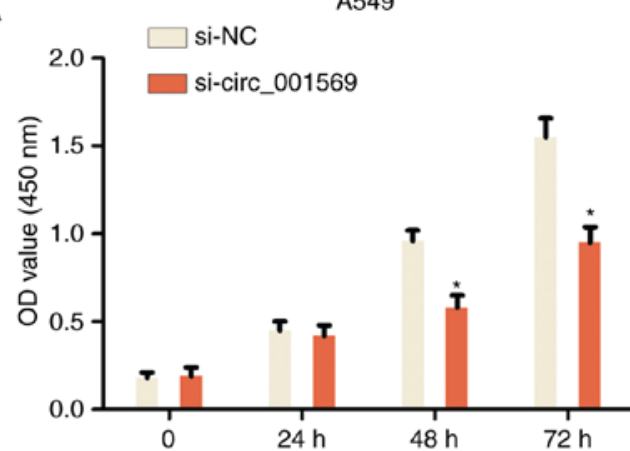

C

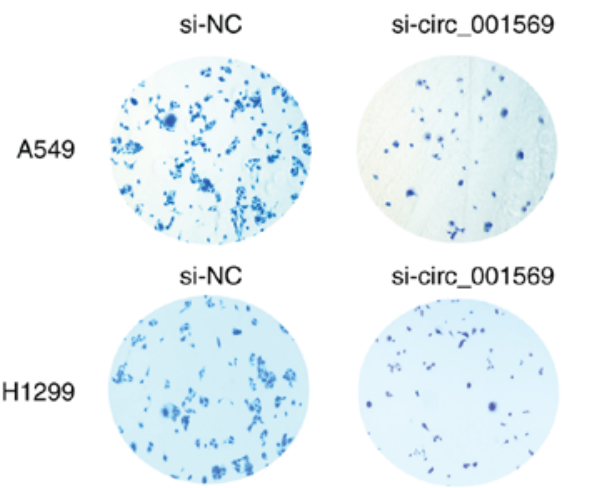

B

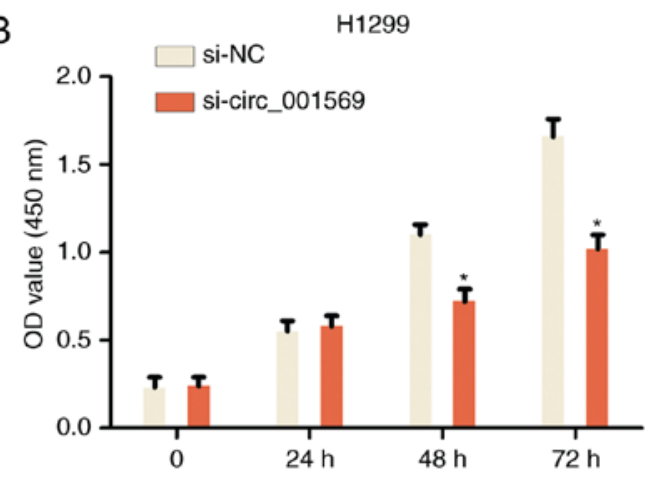

D

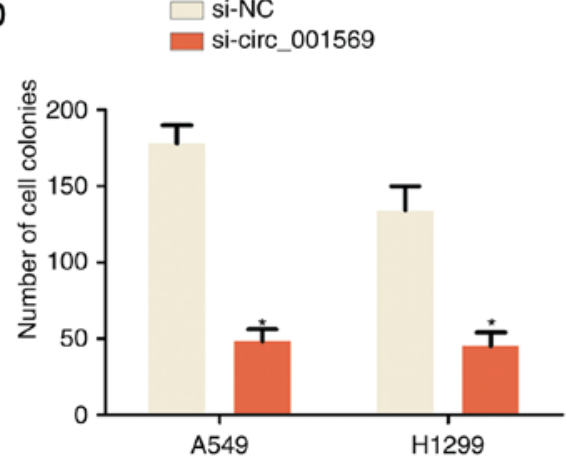

Figure 2. Circ_001569 promotes the proliferation of non-small cell lung cancer cells. A CCK-8 assay was used to evaluate the proliferation rate following the introduction of si-NC or si-circ_001569 into (A) A549 and (B) H1299 cells. P<0.05 vs. si-NC. (C) A cell colony formation assay was conducted and (D) the cell colony number was analyzed following the introduction of si-NC or si-circ_001569 into A549 and H1299 cells compared to si-NC groups. Magnification, $\mathrm{x} 200 .{ }^{*} \mathrm{P}<0.05$. si, small interfering; OD, optical density; NC, negative control. 
A

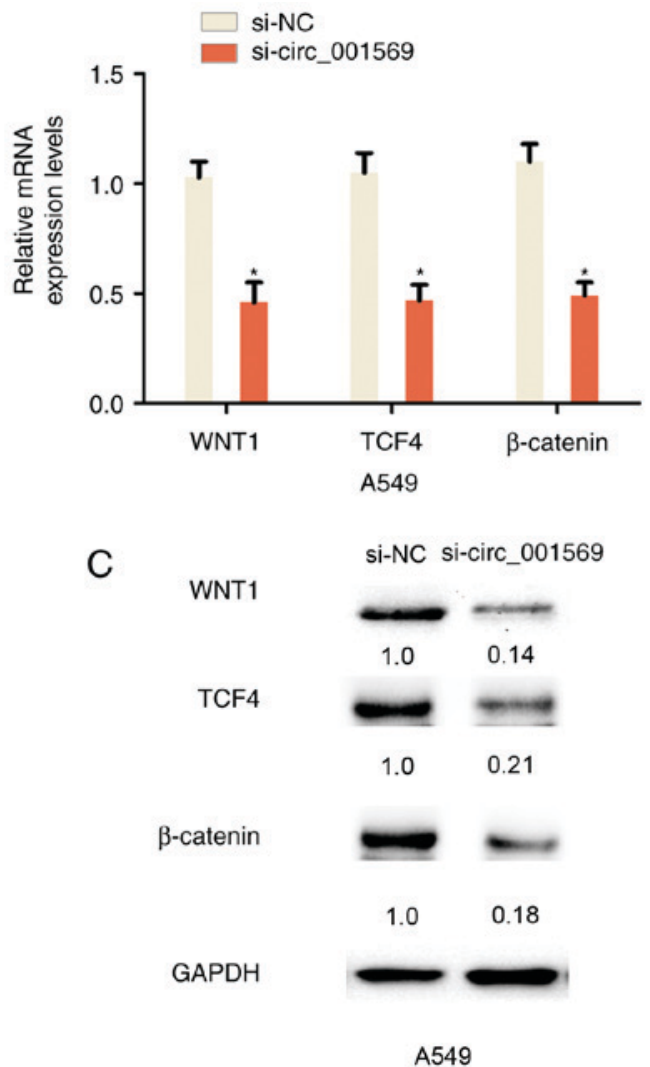

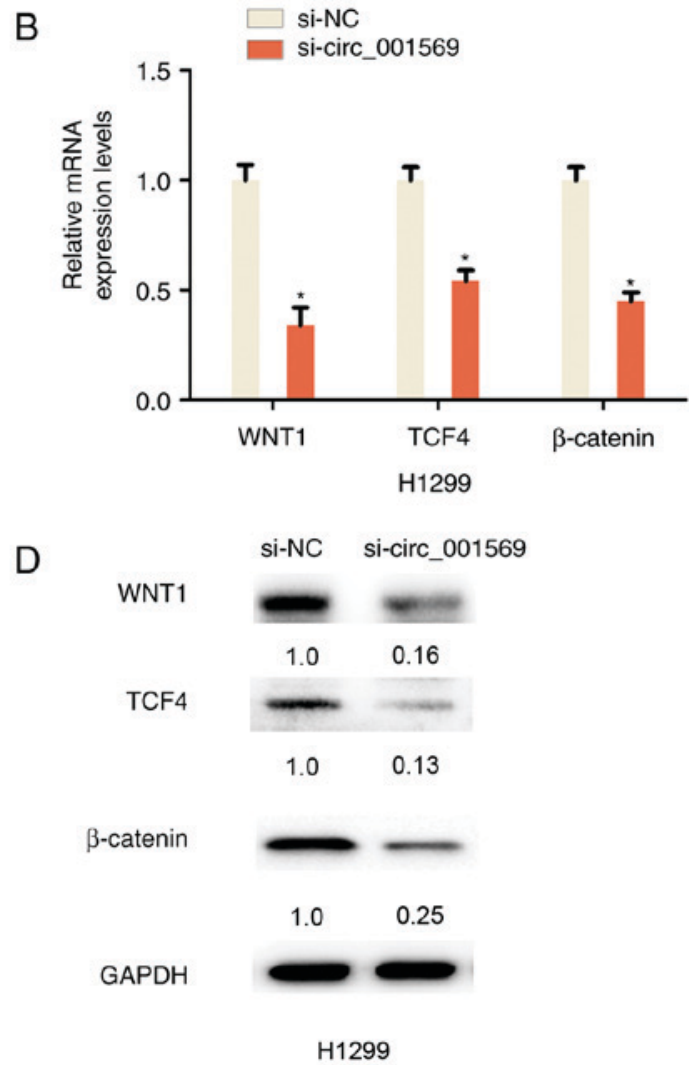

Figure 3. Knockdown of circ_001569 suppressed the Wnt/ 3 -catenin pathway in non-small cell lung cancer cells. Transcription expression levels of WNT1, $\beta$-catenin and TCF4 were measured by reverse transcription quantitative polymerase chain reaction analysis following the introduction of si-NC or si-circ_001569 into (A) A549 and (B) H1299 cells compared to si-NC groups, ${ }^{*} \mathrm{P}<0.05$. Protein expression of WNT1, $\beta$-catenin and TCF4 was measured by western blotting following the introduction of si-NC or si-circ_001569 into (C) A549 and (D) H1299 cells. WNT1, proto-oncogene WNT1; TCF4, transcription factor 4; si, small interfering; NC, negative control.

the knockdown of circ_001569 inhibits the Wnt/ $\beta$-catenin pathway in NSCLC cells.

\section{Discussion}

The majority of the circRNAs have been demonstrated to be exon-containing circular RNAs, and the crucial roles and underlying functions of circRNAs are not well-understood at present $(13,14)$. Certain circRNAs have exhibited significant roles in cancer; for example: hsa_circ_0003159 expression was significantly downregulated in gastric cancer tissues and significantly negatively associated with sex, distal metastasis and TNM stage (15). CircMTO1 suppresses HCC progression by acting as a sponge for oncogenic miR-9 in order to promote cyclin-dependent kinase inhibitor 1 expression, suggesting that circMTO1 is a potential target for HCC treatment (16); hsa_circ_0001895 expression levels were significantly downregulated and correlated with cell differentiation, Borrmann type and tissue carcinoembryonic antigen expression in gastric cancer (17); in NSCLC development and progression, a previous study identified that circRNA_100876 expression levels were demonstrated to be higher in NSCLC tissues and cells and to exhibit a close correlation with lymph node metastasis and TNM stage in NSCLC, In addition, patients with high circRNA_100876 expression exhibited significantly shorter survival rates compared with lower expression groups of patients with NSCLC (18). In the present study, the results indicated that circ_001569 expression was significantly upregulated in NSCLC tissues and cells. Circ_001569 expression was closely associated with differentiation, lymph node metastasis and TNM classification in NSCLC. Patients who exhibited higher circ_001569 expression had a poorer survival outcome when compared with patients with lower circ_001569 expression.

Furthermore, to explore the function of circ_001569 in the development of NSCLC, the present study identified that, following the transfection of A549 and H1299 cells with si-circ_001569, the proliferation rate and cell colony number were significantly reduced, compared with the control group. The Wnt signaling pathway is associated with NSCLC progression, and serine-arginine protein kinase 1 promotes a cancer stem cell-like phenotype through the activation of Wnt/ $\beta$-catenin signaling in NSCLC (19). DEP Domain Containing protein $1 \mathrm{~B}$ enhances cell migration and invasion rate of NSCLC via activation of the $\mathrm{Wnt} / \beta$-catenin signaling pathway (20). Armadillo repeat-containing protein $8 \alpha$ promotes the proliferation and invasion of NSCLC cells by activating the canonical Wnt signaling pathway (21). Knockdown of Homeobox B5 inhibits proliferation, migration and invasion in non-small cell lung cancer cells through inactivation of the Wnt/ $\beta$-catenin pathway (22). The present study demonstrated that the knockdown of circ_001569 inhibited Wnt signaling by reducing the mRNA and protein expression levels of the Wnt signaling pathway-associated genes WNT1, $\beta$-catenin 
and TCF4 in NSCLC cells. In conclusion, the present study demonstrated that circ_001569 expression is higher in NSCLC tissues, and that the knockdown of circ_001569 inhibits proliferation in NSCLC. Furthermore, reduced circ_001569 expression may inhibit the $\mathrm{Wnt} / \beta$-catenin signaling pathway. These results indicate that circ_001569 may present a potential target for NSCLC treatment.

\section{Acknowledgements}

Not applicable.

\section{Funding}

Funding information is not applicable.

\section{Availability of data and materials}

The datasets used and/or analyzed during the current study are available from the corresponding author on reasonable request.

\section{Authors' contributions}

DLC and ZXD made substantial contributions to the conception and design, or acquisition of data, or analysis and interpretation of data. ZXD, YWD, GJ and LJG performed the experiments. ZXD, YWD and GJ drafted the manuscript.

\section{Ethics approval and consent to participate}

The Ethics Board of Nantong Tumor Hospital approved the study. Written informed consent was obtained from all patients.

\section{Consent for publication}

Not applicable.

\section{Competing interests}

The authors declare that they have no competing interests.

\section{References}

1. Siegel RL, Miller KD and Jemal A: Cancer Statistics, 2017. CA Cancer J Clin 67: 7-30, 2017.

2. Torre LA, Bray F, Siegel RL, Ferlay J, Lortet-Tieulent J and Jemal A: Global cancer statistics, 2012. CA Cancer J Clin 65 87-108, 2015.

3. Sanchez de Cos J, Sojo Gonzalez MA, Montero MV, Pérez Calvo MC, Vicente MJ and Valle MH: Non-small cell lung cancer and silent brain metastasis. Survival and prognostic factors. Lung Cancer 63: 140-145, 2009.
4. Meng S, Zhou H, Feng Z, Xu Z, Tang Y, Li P and Wu M: CircRNA: Functions and properties of a novel potential biomarker for cancer. Mol Cancer 16: 94, 2017.

5. He J, Xie Q, Xu H, Li J and Li Y: Circular RNAs and cancer. Cancer Lett 396: 138-144, 2017.

6. Xu L, Zhang M, Zheng X, Yi P, Lan C and Xu M: The circular RNA ciRS-7 (Cdrlas) acts as a risk factor of hepatic microvascular invasion in hepatocellular carcinoma. J Cancer Res Clin Oncol 143: 17-27, 2017.

7. Shang X, Li G, Liu H, Li T, Liu J, Zhao Q and Wang C: Comprehensive circular RNA profiling reveals that hsa circ_0005075, a new circular RNA biomarker, is involved in hepatocellular crcinoma development. Medicine (Baltimore) 95: e3811, 2016.

8. Qin M, Liu G, Huo X, Tao X, Sun X, Ge Z, Yang J, Fan J, Liu L and Qin W: Hsa circ 0001649: A circular RNA and potential novel biomarker for hepatocellular carcinoma. Cancer Biomark 16: 161-169, 2016

9. Xie H, Ren X, Xin S, Lan X, Lu G, Lin Y, Yang S, Zeng Z, Liao W, Ding YQ and Liang L: Emerging roles of circRNA_001569 targeting miR-145 in the proliferation and invasion of colorectal cancer. Oncotarget 7: 26680-26691, 2016.

10. Edge SB and Compton CC: The American Joint Committee on Cancer: The 7th edition of the AJCC cancer staging manual and the future of TNM. Ann Surg Oncol 17: 1471-1474, 2010.

11. Livak KJ and Schmittgen TD: Analysis of relative gene expression data using real-time quantitative PCR and the 2(-Delta Delta C(T)) method. Methods 25: 402-408, 2001.

12. Chen Y, Min L, Ren C, Xu X, Yang J, Sun X, Wang T, Wang F, Sun $C$ and Zhang X: miRNA-148a serves as a prognostic factor and suppresses migration and invasion through Wnt1 in non-small cell lung cancer. PLoS One 12: e0171751, 2017.

13. Qu S, Yang X, Li X, Wang J, Gao Y, Shang R, Sun W, Dou K and Li H: Circular RNA: A new star of noncoding RNAs. Cancer Lett 365: 141-148, 2015.

14. Hansen TB, Kjems J and Damgaard CK: Circular RNA and miR-7 in cancer. Cancer Res 73: 5609-5612, 2013.

15. Tian M, Chen R, Li T and Xiao B: Reduced expression of circRNA hsa_circ_0003159 in gastric cancer and its clinical significance. J Clin Lab Anal 32, 2018.

16. Han D, Li J, Wang H, Su X, Hou J, Gu Y, Qian C, Lin Y, Liu X, Huang M, et al: Circular RNA circMTO1 acts as the sponge of microRNA-9 to suppress hepatocellular carcinoma progression. Hepatology 66: 1151-1164, 2017.

17. Shao Y, Chen L, Lu R, Zhang X, Xiao B, Ye G and Guo J: Decreased expression of hsa_circ_0001895 in human gastric cancer and its clinical significances. Tumour Biol 39: 1010428317699125, 2017.

18. Yao JT, Zhao SH, Liu QP, Lv MQ, Zhou DX, Liao ZJ and Nan KJ: Over-expression of CircRNA_100876 in non-small cell lung cancer and its prognostic value. Pathol Res Pract 213: 453-456, 2017.

19. Gong L, Song J, Lin X, Wei F, Zhang C, Wang Z, Zhu J, Wu S, Chen Y, Liang J, et al: Serine-arginine protein kinase 1 promotes a cancer stem cell-like phenotype through activation of Wnt/ $\beta$-catenin signalling in NSCLC. J Pathol 240: 184-196, 2016.

20. Yang Y, Liu L, Cai J, Wu J, Guan H, Zhu X, Yuan J and Li M: DEPDC1B enhances migration and invasion of non-small cell lung cancer cells via activating Wnt $/ \beta$-catenin signaling. Biochem Biophys Res Commun 450: 899-905, 2014.

21. Xie C, Jiang G, Fan C, Zhang X, Zhang Y, Miao Y, Lin X, Wu J, Wang L, Liu Y, et al: ARMC8 $\alpha$ promotes proliferation and invasion of non-small cell lung cancer cells by activating the canonical Wnt signaling pathway. Tumour Biol 35: 8903-8911, 2014.

22. Zhang B, Li N and Zhang H: Knockdown of homeobox B5 (HOXB5) inhibits cell proliferation, migration, and invasion in non-small cell lung cancer cells through inactivation of the Wnt/ $\beta$-catenin pathway. Oncol Res 26: 37-44, 2018. 